\title{
Front Matter: Volume 11312
}

, "Front Matter: Volume 11312," Proc. SPIE 11312, Medical Imaging 2020: Physics of Medical Imaging, 1131201 (29 May 2020); doi:

$10.1117 / 12.2570912$

SPIE. Event: SPIE Medical Imaging, 2020, Houston, Texas, United States 


\title{
PROGRESS IN BIOMEDICAL OPTICS AND IMAGING
}

\section{Medical Imaging 2020 \\ Physics of Medical Imaging}

\author{
Guang-Hong Chen \\ Hilde Bosmans \\ Editors
}

16-19 February 2020

Houston, Texas, United States

Sponsored by

SPIE

Cooperating Organizations

AAPM-American Association of Physicists in Medicine (United States)

MIPS-Medical Image Perception Society (United States)

SIIM-Society for Imaging Informatics in Medicine (United States)

IFCARS—International Foundation for Computer Assisted Radiology and Surgery (Germany)

WMIS—World Molecular Imaging Society

Published by

SPIE

\section{Volume 11312}

Part One of Two Parts 
The papers in this volume were part of the technical conference cited on the cover and title page. Papers were selected and subject to review by the editors and conference program committee. Some conference presentations may not be available for publication. Additional papers and presentation recordings may be available online in the SPIE Digital Library at SPIEDigitalLibrary.org.

The papers reflect the work and thoughts of the authors and are published herein as submitted. The publisher is not responsible for the validity of the information or for any outcomes resulting from reliance thereon.

Please use the following format to cite material from these proceedings:

Author(s), "Title of Paper," in Medical Imaging 2020: Physics of Medical Imaging, edited by GuangHong Chen, Hilde Bosmans, Proceedings of SPIE Vol. 11312 (SPIE, Bellingham, WA, 2020) Seven-digit Article CID Number.

ISSN: 1605-7422

ISSN: 2410-9045 (electronic)

ISBN: 9781510633919

ISBN: 9781510633926 (electronic)

Published by

SPIE

P.O. Box 10, Bellingham, Washington 98227-0010 USA

Telephone +1 3606763290 (Pacific Time) · Fax +1 3606471445

SPIE.org

Copyright (c) 2020, Society of Photo-Optical Instrumentation Engineers.

Copying of material in this book for internal or personal use, or for the internal or personal use of specific clients, beyond the fair use provisions granted by the U.S. Copyright Law is authorized by SPIE subject to payment of copying fees. The Transactional Reporting Service base fee for this volume is $\$ 21.00$ per article (or portion thereof), which should be paid directly to the Copyright Clearance Center (CCC), 222 Rosewood Drive, Danvers, MA 01923. Payment may also be made electronically through CCC Online at copyright.com. Other copying for republication, resale, advertising or promotion, or any form of systematic or multiple reproduction of any material in this book is prohibited except with permission in writing from the publisher. The CCC fee code is $1605-$ $7422 / 20 / \$ 21.00$.

Printed in the United States of America by Curran Associates, Inc., under license from SPIE.

Publication of record for individual papers is online in the SPIE Digital Library.

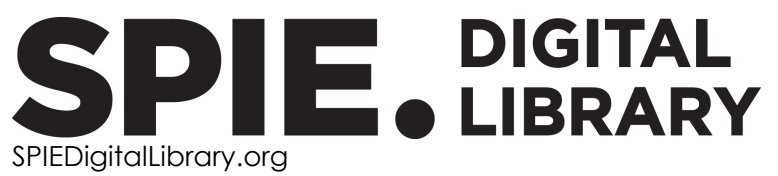

Paper Numbering: Proceedings of SPIE follow an e-First publication model. A unique citation identifier (CID) number is assigned to each article at the time of publication. Utilization of CIDs allows articles to be fully citable as soon as they are published online, and connects the same identifier to all online and print versions of the publication. SPIE uses a seven-digit CID article numbering system structured as follows:

- The first five digits correspond to the SPIE volume number.

- The last two digits indicate publication order within the volume using a Base 36 numbering system employing both numerals and letters. These two-number sets start with $00,01,02,03,04$, 05, 06, 07, 08, 09, 0A, OB ... 0Z, followed by 10-1Z, 20-2Z, etc. The CID Number appears on each page of the manuscript. 


\title{
Contents
}

\author{
xvii Authors \\ xxiii Conference Committee \\ xxvii 2020 Medical Imaging Award Recipients
}

\section{Part One}

\section{NEW TECHNOLOGIES IN IMAGING}

1131202 A new concept for fluence field modulation in x-ray CT: the z-sbDBA [11312-2]

$1131203 \quad \mathrm{C}$-arm CT imaging using the extended line-ellipse-line trajectory: seamless FBP reconstruction from real data [1 1312-3]

1131204 Preliminary imaging evaluation of a compact tomosynthesis system for potential point-of-care extremity imaging [1 1312-4]

1131205 Slot-scan dual-energy measurement of bone mineral density on a robotic x-ray system [11312-5]

\section{CT RECONSTRUCTION WITH DL TECHNIQUES}

1131206 Deep learning enabled prior image constrained compressed sensing (DL-PICCS) reconstruction framework for sparse-view reconstruction [11312-6]

1131207 Generalized iterative sparse-view CT reconstruction with deep neural network [11312-7]

1131208 A deep learning reconstruction method for sparsely spaced multidetector helical CT using cascaded dual-domain CNN [11312-8]

$1131209 \quad$ Ultra-fast-pitch acquisition and reconstruction in helical CT [1 1312-9]

$113120 \mathrm{~A}$ Fast spectral x-ray CT reconstruction with data-adaptive, convolutional regularization [11312-10]

11312 OB Semi-supervised learned sinogram restoration network for low-dose CT image reconstruction [11312-11] 
$113120 \mathrm{C}$ Multi-energy inter-pixel coincidence counters for charge sharing correction and compensation for photon counting detectors: assessment at a normalized spatial resolution [11312-12]

$113120 D$ A study of energy resolution in CPD indirect photon-counting x-ray imaging [11312-13]

$11312 \mathrm{OE} \quad 1 \boldsymbol{\mu m}$ spatial resolution in silicon photon-counting $\mathrm{CT}$ detectors by measuring charge diffusion [11312-14]

$11312 \mathrm{OF} \quad$ Hybrid energy integrating and photon counting micro-CT [11312-15]

$113120 \mathrm{G}$ Performance of amorphous selenium-based unipolar charge sensing detector for photon counting $x$-ray imaging [11312-16]

\section{QUALITY ASSESSMENT AND OPTIMIZATION IN BREAST IMAGING}

$11312 \mathrm{OH} \quad$ Factors affecting microcalcification detection of wide-angle digital breast tomosynthesis and strategies for improving performance [11312-17]

1131201 Determining the optimal angular range of the $x$-ray source motion in tomosynthesis using virtual clinical trials [11312-18]

$113120 \mathrm{~J}$ Evaluation of possible phantoms for assessment of image quality in synthetic mammograms [11312-19]

$113120 \mathrm{~K}$ Equivalent breast thickness and dose sensitivity of a next iteration 3D structured breast phantom with lesion models [11312-20]

$113120 \mathrm{~L} \quad$ High resolution microcalcification signal profiles for dedicated breast CT [11312-21]

$113120 \mathrm{M}$ Classification of breast calcifications in dual-energy FFDM using a convolutional neural network: simulation study [11312-22]

\section{BREAST IMAGING: NEW TECHNOLOGY}

$1131200 \quad$ Volumetric breast density estimation using dual energy digital breast tomosynthesis [11312-24]

$11312 \mathrm{OP} \quad$ An investigation of slot-scanning for mammography and breast CT [11312-25]

$113120 Q \quad$ Deep convolutional neural network denoising for digital breast tomosynthesis reconstruction [11312-26] 
11312 OR Modelling the use of stationary, rectangular arrays of x-ray emitters for digital breast tomosynthesis [1 1312-27]

\section{INNOVATIONS IN NUCLEAR MEDICINE AND MRI}

11312 OT An assessment of PET dose reduction with penalized likelihood image reconstruction using a computationally efficient model observer [11312-29]

$113120 \mathrm{U} \quad$ Joint low-count PET/CT segmentation and reconstruction with paired variational neural networks [11312-30]

$113120 \mathrm{~V} \quad$ Medical image reconstruction with image-adaptive priors learned by use of generative adversarial networks [11312-31]

$113120 \mathrm{~W} \quad$ Quality assessment of brain MRI scans using a dense neural network model and image metrics [1 1312-32]

\section{DETECTOR TECHNOLOGIES}

$113120 \mathrm{X}$ Initial characterization of a hybrid direct-indirect active matrix flat panel imager for digital radiography [11312-33]

11312 OY The effect on patient dose and image quality of simultaneous acquisition of high-resolution small field of view (FOV) and regular-resolution large FOV imaging modes of a dual-resolution imaging detector [11312-34]

$113120 Z \quad$ Analogous Lubberts effect in photon counting detectors [1 1312-35]

1131210 Theoretical performance of polycrystalline mercuric iodide x-ray converters incorporating pillar-supported Frisch grid structures [11312-36]

$1131211 \quad$ Novel irradiation side sampling system flexible flat panel detectors with high image quality and light weight [1 1312-37]

$1131212 \quad$ Pixelated columnar CsI:TI scintillator for high resolution radiography and cone-beam CT [11312-38]

TARGET: CONTRAST AGENT IMAGING OPTIMIZED

1131214 Patient-informed modelling of hepatic contrast dynamics in contrast-enhanced CT imaging [1 1312-40]

1131215 Contrast flow velocity quantification from time-resolved CT angiography: a phantom study [1 1312-41] 
1131216 Power injector for angiographic flow analysis using custom contrast density profiles [11312-42]

1131217 Simulation of contrast agent dynamics in digital brain phantom for CT perfusion optimization [11312-43]

1131218 Combining spectral CT acquisition methods for high-sensitivity material decomposition [11312-44]

\section{X-RAY PHASE CONTRAST IMAGING}

$113121 \mathrm{~A} \quad$ A preclinical large-field-of-view x-ray multi-contrast lung imaging prototype [11312-46]

$113121 \mathrm{~B} \quad$ Phase contrast $\mathrm{CT}$ enabled three-material decomposition in spectral CT imaging (Second Place: Robert F. Wagner Best Student Paper Award and Physics of Medical Imaging Student Paper Award) [11312-47]

$113121 \mathrm{C} \quad$ Low dose and high SNR phase retrieval and micro CT using a wide area photon counting detector [11312-48]

$113121 \mathrm{E} \quad$ Reducing the $\mathrm{x}$-ray phase contrast image bias via deep computed signal estimation technique [11312-50]

\section{PHOTON COUNTING CT APPLICATIONS}

$113121 \mathrm{~F} \quad$ Spectral CT metal artifact reduction using weighted masking and a one step direct inversion reconstruction algorithm [11312-51]

$113121 \mathrm{G} \quad$ Bone density quantification via material decomposition in an arthritic mouse model using photon counting spectral CT [11312-52]

$113121 \mathrm{H} \quad$ A new series expansion method and its application to photon-counting $\mathrm{CT}$ reconstruction [11312-53]

1131211 Development of a spectral micro-CT system using a photon counting detector with anti-coincidence corrections [11312-54]

$113121 \mathrm{~J}$ Impact of photon counting detector spectral distortion on virtual non-contrast CT imaging [11312-55]

\section{CBCT RECONSTRUCTION TECHNIQUES}

$113121 \mathrm{~K} \quad$ High temporal resolution time-resolved C-arm cone-beam CT imaging using a multi-sweep data acquisition scheme [11312-56]

$113121 \mathrm{~L} \quad$ Deep learning-aided $\mathrm{CBCT}$ image reconstruction of interventional material from four $\mathrm{x}$-ray projections [11312-57] 
$113121 \mathrm{M}$ Estimation of local deformable motion in image-based motion compensation for interventional cone-beam CT [11312-58]

$113121 \mathrm{~N}$ An improved combination of ordered subsets and momentum for fast model-based iterative CT reconstruction [1 1312-59]

\section{SPECTRAL CT NEW HARDWARE}

1131210 Dual layer x-ray detector simulation [11312-60]

$113121 \mathrm{P} \quad$ Spectral modulator with flying focal spot for cone-beam CT: a feasibility study [11312-61]

$113121 Q \quad$ Benefit of dual energy CT for lesion localization and classification with convolutional neural networks [11312-62]

11312 IR Multi-energy CT with triple x-ray beams: a feasibility animal study [11312-63]

$1131215 \quad$ Accurate proton stopping power images reconstructed using joint statistical dual energy CT: experimental verification and impact of fan-beam CT scatter [11312-64]

VIRTUAL IMAGING

$113121 \mathrm{~T} \quad$ Virtual imaging trials: an emerging experimental paradigm in imaging research and practice [11312-65]

$113121 \mathrm{U}$ In vivo noise texture estimation: development and validation of an automated methodology [11312-66]

$113121 \mathrm{~V} \quad$ High-spatial-resolution Monte Carlo simulations of small-animal x-ray fluorescence tomography [11312-67]

$113121 \mathrm{~W}$ Simulation of hepatic arteries and synthesis of 2D fluoroscopic images for interventional imaging studies [11312-68]

$113121 \mathrm{Y}$ Optimization of energy thresholds in photon-counting CT via a virtual clinical trial [11312-70]

\section{NEW RECONSTRUCTION TECHNIQUES IN DUAL ENERGY CT}

1131212 Prospective prediction and control of image properties in model-based material decomposition for spectral CT [11312-71]

1131220 Comparative study of dual energy cone-beam CT using a dual-layer detector and kVp switching for material decomposition [1 1312-72] 
$1131221 \quad$ Tensor convolutional neural network architecture for spectral CT reconstruction [11312-73]

1131222 Feasibility of achieving spectral CT imaging from a single KV acquisition and deep learning method [11312-74]

1131223 A cascaded deep-learning reconstruction method for sparse-view kV-switching dual-energy CT [1 1312-75]

\section{ARTIFACTS IN CBCT SOLVED}

1131224 Evaluation of patient-specific scatter-corrected digital chest tomosynthesis [11312-76]

1131225 Projection-domain metal artifact correction using a dual layer detector [11312-77]

1131226 Method for metal artifact avoidance in C-arm cone-beam CT [11312-78]

$1131227 \quad$ Non-circular CT orbit design for elimination of metal artifacts [11312-79]

1131228 Reduction of ring artifacts caused by 2D anti-scatter grids in flat-panel CBCT [11312-80]

POSTER SESSION: IMAGING METHODS INCLUDING OPTICAL, MR, AND ULTRASOUND

$113122 \mathrm{~B} \quad$ Calibration method of conformal $x$-ray transmission imaging system based on small ball phantom [11312-82]

$113122 \mathrm{C} \quad$ Artifact correction for small metals by combining pattern matching and interpolation methods in x-ray [1 1312-83]

11312 2D Effect of geometric variations on image characteristics in digital tomosynthesis [11312-84]

$113122 \mathrm{E} \quad$ Multi-beam x-ray sources with carbon nanotube emitter for tomosynthesis system [1 1312-85]

$113122 \mathrm{~F} \quad$ High-resolution vascular imaging of small animal using the NIR-Ilb window emitted from ICG [1 1312-86]

$113122 \mathrm{G} \quad$ Bone health assessment using synthetic aperture ultrasound reflectometry [11312-87]

$113122 \mathrm{H} \quad$ A finite element mesh regrouping-based hybrid light transport model for enhancing the efficiency and accuracy in bioluminescence tomography [11312-88]

$113122 \mathrm{~J} \quad$ Keratoconus stage impact on visual acuity and contrast sensitivity [11312-90]

$113122 \mathrm{~K}$ 4D image construction from free-breathing MRI slice acquisitions of the thorax based on a concept of flux [1 1312-91] 
POSTER SESSION: MEASUREMENT METHODS

$113122 \mathrm{M} \quad$ Reconstruction of $x$-ray focal spot distribution using a rotating edge [11312-93]

$113122 \mathrm{~N} \quad$ Image quality assessment of real patient thorax CT images using modulation transfer function and noise power spectrum [11312-94]

1131220 A method to assess the performance and the relevance of segmentation in radiomic characterization [1 1312-95]

$113122 \mathrm{~A} \quad$ Automatic phantom test pattern classification through transfer learning with deep neural networks [11312-97]

POSTER SESSION: CONE BEAM CT

$113122 R \quad$ Displaying information collected by intraoral tomosynthesis as multi-view synthetic radiographs [11312-98]

1131225 3D-localization of anatomic structures in tomographic images from optical flow of projection images [11312-99]

$113122 \mathrm{~T} \quad$ Implementation, validation and application of a tool for the assessment of the modulation transfer function and noise power spectrum of dental CBCT scanners [1 1312-100]

$113122 \mathrm{U} \quad$ Synthesis of ghost-free panoramic radiographs from dental CBCT images [11312-101]

\section{Part Two}

$113122 \mathrm{~V} \quad$ Micro computed tomography system based on field emission x-ray source with carbon nanotube emitter [11312-103]

11312 2W Simultaneous scatter rejection and correction method using 2D antiscatter grids for CBCT [11312-104]

$113122 \mathrm{X} \quad$ Projection-based adaptive scatter correction for CT system [11312-105]

POSTER SESSION: WORK INVOLVING DEVELOPMENT OF PHANTOMS OR ANATOMICAL SIMULATION METHODS

$113122 Y \quad$ CT phantom with 3D anthropomorphic, contrast-enhanced texture [11312-106]

$113122 Z$ Prediction of pleural invasion of lung cancer with dynamic chest radiography: a simulation study [11312-107] 
1131230 Quantifying motion artifacts using a rotating phantom: insights towards dynamic musculoskeletal applications [11312-108]

1131231 Quantitative evaluation of cadaveric contrast agents: identifying anatomical structures with BriteVu [1 1312-109]

$1131232 \quad$ Impact of chromophores on colour appearance in a computational skin model [11312-110]

POSTER SESSION: NEUROIMAGING

1131233 Fast and accurate brain $\mathrm{T}_{2}$ relaxation time quantification in animal models calibrated using gel phantoms and in vivo data suitable for imaging at a biosafety level 4 environment [ $11312-1111]$

1131234 Physical image simulation of human brain in case of acute stroke [11312-112]

1131235 Effect of truncated singular value decomposition on digital subtraction angiography derived angiographic parametric imaging maps [11312-113]

$1131236 \quad$ Non-contrast head CT-assisted cerebral CT perfusion imaging [11312-114]

$1131237 \quad$ Feasibility of a stationary head CT scanner using a CNT x-ray source array [11312-115]

1131238 Simulation and optimization of system configuration for the stationary head CT using CNT x-ray source array: reconstruction and quality evaluation [11312-116]

1131239 Quantification of fiber orientation uncertainty in polarized light imaging of the human brain [11312-117]

POSTER SESSION: IMAGE GUIDED INTERVENTIONS

$113123 \mathrm{~A}$ Comparison of the patient's skin dose for flat and curved surfaces as a function of x-ray beam angle of incidence [11312-118]

$113123 B \quad$ Evaluation of geometric and exposure parameters used in fluoroscopically-guided neuro-interventional procedures [11312-119]

$113123 \mathrm{C}$ Methods for object tracking and shadowing in a top-down view virtual reality scattered radiation display system (SDS) for fluoroscopically-guided procedures [11312-120]

$113123 D$ Considerations for accurate inclusion of staff member body tracking in a top-down view virtual reality display of a scattered radiation dose map during fluoroscopic interventional procedures [11312-121]

$113123 \mathrm{E} \quad$ Variation of eye-lens dose with variation of the location of the beam isocenter in the head during neuro-interventional fluoroscopic procedures [11312-122] 
$113123 \mathrm{~F} \quad$ Adaptively variable frame-rate fluoroscopy with an ultra-fast digital $\mathrm{x}$-ray tube based on carbon nanotube field electron emitters [11312-123]

$113123 \mathrm{G}$ Quantitative blood flow imaging with time-resolved C-arm cone-beam CT imaging [11312-124]

$113123 \mathrm{H} \quad$ Assessment of proton radiographic sensitivity limits for gold nanoparticle tagged tumors using gold leaf phantoms [11312-125]

$1131231200 \mathrm{kV} x$-ray source for radiotherapy and imaging: preliminary results and discussion [11312-126]

$113123 \mathrm{~J} \quad$ Nullspace-constrained modifications of under-sampled interventional CT images using instrument-specific prior information [11312-127]

POSTER SESSION: IMAGE RECONSTRUCTION INCLUDING CT, SPECT, PET, OCT, AND TOMOSYNTHESIS

$113123 \mathrm{~K}$ Accelerated reconstruction for inverse geometry CT via derivative back-projection filtration [11312-128]

$113123 \mathrm{~L} \quad$ Artifacts reduction in 4D-CBCT via a joint free-form registration method of projection match and gradient constraint [11312-129]

$113123 \mathrm{M}$ A stopping criterion for iterative reconstruction of x-ray computed tomography [11312-130]

$113123 \mathrm{~N} \quad$ Micro-CT imaging of super-resolution MBIR algorithm based on sub-pixel displacement [11312-131]

1131230 Low-dose digital tomosynthesis reconstruction based on field emission flat-panel x-ray source array [11312-132]

$113123 P \quad$ Automatic geometric calibration in 3D parallel geometry [11312-133]

$113123 Q \quad$ Model-based material decomposition with system blur modeling (Cum Laude Poster Award) [11312-134]

11312 3R Rotating projection based localizer radiograph which enables multiple pre-scan function for CT [11312-135]

1131235 Single scan dual energy cone beam CT using a rotating filter [11312-136]

11312 3T Average consistency: a superior way of using the composite image to boost dynamic CT reconstruction [11312-137]

POSTER SESSION: DETECTOR TECHNOLOGY: SCINTILLATORS, PHOTOCONDUCTORS, DIODES, AND TFT 
$113123 X \quad$ The effects of $x$-ray irradiation on a-IGZO TFTs used for active pixel sensor [11312-141]

$113123 Y \quad$ Response of CZT pixels to parallel and oblique x-rays [11312-142]

$113123 Z$ Extended dynamic range CMOS active pixel architecture for x-ray detectors [11312-143]

\section{POSTER SESSION: MACHINE LEARNING APPLIED TO IMAGING PHYSICS}

1131240 Combined spatial and temporal deep learning for image noise reduction of fluoroscopic x-ray sequences [11312-145]

$1131241 \quad$ Low-dose CT reconstruction with simultaneous sinogram and image domain denoising by deep neural network [11312-146]

$1131242 \quad$ Progressive transfer learning strategy for low-dose CT image reconstruction with limited annotated data [11312-147]

1131243 Deep neural networks for low-dose CT image reconstruction via cooperative meta-learning strategy [11312-148]

1131244 Semi-supervised noise distribution learning for low-dose CT restoration [1 1312-149]

1131245 Simultaneous denoising and spatial resolution enhancement using convolutional neural network-based linear model in diagnostic CT images [1 1312-150]

1131246 Construction of virtual normal dose CT images from ultra-low dose CT images using dilated residual networks [11312-151]

1131247 Low dose PET imaging with CT-aided cycle-consistent adversarial networks [11312-152]

1131248 Attenuation correction for PET/MRI using MRI-based pseudo CT [11312-153]

1131249 PET attenuation correction using non-AC PET-based synthetic CT [1 1312-154]

$113124 \mathrm{~A} \quad$ Prior knowledge driven machine learning approach for PET sinogram data denoising [11312-155]

$113124 \mathrm{~B} \quad$ Non-local texture learning approach for CT imaging problems using convolutional neural network [11312-156]

11312 4C Dual-energy CT reconstruction using deep mutual-domain knowledge for basis decomposition and denoising [11312-157]

$113124 D \quad$ Unsupervised data fidelity enhancement network for spectral CT reconstruction [11312-158]

11312 4E Pixel-defect corrections for radiography detectors based on deep learning [1 1312-159] 
$113124 \mathrm{~F}$ Development of a denoising convolutional neural network-based algorithm for metal artifact reduction in digital tomosynthesis [11312-160]

$113124 \mathrm{H} \quad$ Correction for cone beam CT image artifacts via a deep learning method [11312-162]

1131241 Reduction of truncation artifact in stationary inverse-geometry digital tomosynthesis using convolutional neural network [11312-163]

$113124 \mathrm{~J}$ Iterative reconstruction of cone-beam breast CT using plug-and-play projected gradient descent [11312-164]

$113124 \mathrm{~L} \quad$ Deep learning-based relative stopping power mapping generation with cone-beam CT in proton radiation therapy [11312-166]

$113124 \mathrm{M}$ Stopping power map estimation from dual-energy CT using deep convolutional neural network [11312-167]

$113124 \mathrm{~N} \quad$ A deep RNN for CT image reconstruction [11312-168]

1131240 Evaluation of deep learning segmentation for rapid, patient-specific CT organ dose estimation using an LBTE solver [11312-169]

$113124 \mathrm{P} \quad$ Deep learning-based low dose CT imaging [11312-170]

$113124 Q$ Use of a convolutional neural network (CNN) to determine if the patient's eye lens is in the beam for $\mathrm{X}$-ray image projections [11312-171]

POSTER SESSION: PHOTON COUNTING IMAGING

$113124 R \quad$ A blooming correction technique for improved vasa vasorum detection using an ultra-high-resolution photon-counting detector CT [11312-172]

$113124 S$ Sparse view-CT based on photon counting detector using multi-level wavelet-CNN [11312-173]

$113124 \mathrm{~T}$ The effects of the spatio-energetic system response of cadmium telluride $\mathrm{x}$-ray detectors on basis-material decomposition for iodine imaging tasks [11312-174]

$113124 \mathrm{U} \quad$ Leveraging deep generative model for direct energy-resolving $\mathrm{CT}$ imaging via existing energy-integrating $\mathrm{CT}$ images [11312-175]

$113124 \mathrm{~V}$ Development of 3-material decomposition method combining multiple 2-material decompositions in photon counting CT [11312-176]

$113124 \mathrm{~W}$ Three material decomposition for spectral imaging without contrast agents in photon-counting CT-modeling and feasibility study [11312-177]

$113124 \mathrm{X}$ Optimization of basis material selection and energy binning in three material decomposition for spectral imaging without contrast agents in photon-counting CT [11312-178] 
$113124 \mathrm{Y} \quad$ Development of a benchtop photon counting cone-beam CT system with a translate-rotate geometry [11312-179]

\section{POSTERS SESSION: PHASE CONTRAST IMAGING}

$113124 Z$ Investigation of artifacts due to large-area grating defects and correction using short window Fourier transform and convolution neural networks for phase-contrast $x$-ray interferometry [11312-180]

1131250 Comparison of the phase and absorption contrast tomographic reconstruction [11312-181]

$1131251 \quad$ A comparison of phase retrieval methods for propagation-based contrast $\mathbf{x}$-ray imaging with polychromatic sources [11312-182]

1131252 Objective characterization of an in-line phase sensitive imaging prototype using a mid-energy beam [11312-183]

1131253 Development of simulations for a mesh-based x-ray phase imaging system [11312-184]

1131255 Phantom measurements with a mesh-based phase system employing polycapillary optics [11312-186]

\section{POSTER SESSION: MAMMOGRAPHY}

1131256 A prototype system of upright dedicated cone-beam breast CT [11312-188]

1131257 Pre-processing for image quality improvement in simultaneous DBT and mechanical imaging [11312-189]

1131258 Evaluation of digital breast tomosynthesis systems [11312-190]

1131259 Virtual clinical trials in 3D and 2D breast imaging with digital phantoms derived from clinical breast CT scans [11312-191]

$113125 \mathrm{~A} \quad$ Quantitative evaluation of virtual monochromatic imaging for detection of microcalcifications [11312-192]

$113125 \mathrm{~B} \quad$ Spectral signatures from small angle $\mathbf{x}$-ray scattering for breast cancer discrimination [11312-193]

$113125 \mathrm{C}$ Tabletop high-resolution breast specimen imaging system based on field emission CNT x-ray source [11312-194]

$113125 D$ Preliminary data on TruviewMAG to assess the visibility of microcalcifications and image quality of mammograms: comparison with the standard magnification view [11312-195] 
$113125 \mathrm{E} \quad$ Early results for equivalent wavefield transform as a direct solution to the inverse modeling problem for active infrared thermography and potential for perfusion information to differentiate healthy versus cancerous breast tissue [11312-196]

11312 5F Advancing synthetic mammography for stationary digital breast tomosynthesis [11312-197]

$113125 \mathrm{G} \quad$ Proposing rapid source pulsing for improved super-resolution in digital breast tomosynthesis [11312-198]

$113125 \mathrm{H} \quad$ 3D redatuming for breast ultrasound [11312-199]

$1131251 \quad$ Deep learning model observer for 4-alternative forced choice in digital breast tomosynthesis [1 1312-200]

$113125 \mathrm{~J} \quad$ Effect of tissue density on the temperature pattern of the breast [11312-201] 
Proc. of SPIE Vol. 11312 1131201-16

Downloaded From: https://www.spiedigitallibrary.org/conference-proceedings-of-spie on 25 Apr 2023 Terms of Use: https://www.spiedigitallibrary.org/terms-of-use 


\section{Authors}

Numbers in the index correspond to the last two digits of the seven-digit citation identifier (CID) article numbering system used in Proceedings of SPIE. The first five digits reflect the volume number. Base 36 numbering is employed for the last two digits and indicates the order of articles within the volume. Numbers start with 00, 01, 02, 03, 04, 05, 06, 07, 08, 09, OA, OB...0Z, followed by 10-1Z, 20-2Z, etc.

Abadi, Ehsan, 14, 1T, 1U, 1Y, $2 Z$

Abbey, Craig K., OL

Acciavatti, Raymond J., Ol, 5G

Adamson, Philip M., 40

Aerden, Dimitri, 15

Ahn, Chulkyun, $2 \mathrm{U}$

Ahn, Jung Sun, 2E, 5C

Ahn, Yujung, 3F

Akamatsu, Keiichi, 11

Albarqouni, Shadi, 1Q

Allman, Ariana B., 35

Allmendinger, Thomas, $1 \mathrm{R}$

Altunbas, Cem, 2W

Amin, Mitesh, 2G

Amunts, Katrin, 39

Anastasio, Mark A., OV

Anderson, Jill, 4R

Anthony, Brian W., 2G

Antonuk, Larry E., 10

Arnab, Salman M., OG

Aulwes, Ethan F., 3H

Axer, Markus, 39

Baba, Hiroyasu, OD

Badal, Andreu, OM

Badea, C. T., OA, OF, 11

Bader, A. M., 4Y

Bakic, Predrag R., 0l, 32, 57

Bambot, Shabbir, OP

Barber, Rina Foygel, $1 F$

Barufaldi, Bruno, Ol, 5G

Bazalova-Carter, Magdalena, 3

Beck, Stefanie, 1Q

Becker, Amy E., OL

Bednarek, Daniel R., OY, 3A, 3B, 3C, 3D, 3E, 4Q

Beilter, Jonathan J., 4M

Bennett, N. Robert, 1L, 1P, 20, 25, 2M

Beuing, Oliver, $2 S$

Bhadra, Sayantan, OV

Bian, Zhaoying, OB, 42, 43, 44, 4B, 4D, 4U

Billingsley, Alex J., 04, 24, 37

Bliznakova, Kristina, 59

Bollinger, Laura, 33

Boone, John M., OL, 3I, 59

Boonen, Pieter Thomas, 15

Bosmans, Hilde, OJ, OK, 2T

Boyd, Douglas, 31

Brankov, Jovan G., 50

Brown, Conner, 12

Buls, Nico, 15, 30
Bushe, Dan, 22

Buzzatti, Luca, 30

Byrum, Russell, 33

Cahill, Patrick J., 2K

Cai, Jianmei, 30

Cai, Meishan, $2 \mathrm{~F}$

Camlica, Ahmet, OG

Cao, Qian, 3Q

Capostagno, S., $1 \mathrm{M}$

Castro, Marcelo A., 33

Cattrysse, Erik, 30

Chan, Heang-Ping, $0 Q$

Chang, Jung Min, 5D

Chen, Gaofeng, 44

Chen, Guang-Hong, 06, 1B, 1J, 1K, 22, 3G, 4H

Chen, Hong, 31

Chen, Jianwei, $1 \mathrm{E}$

Chen, Wenhao, 3R

Chen, Yang, 4C

Chen, Zhiqiang, $1 \mathrm{~A}$

Cheung, Pikting, 55

Cho, Seungryong, 2E

Choi, Jae Young, 4E

Choi, Jinho, 5C

Choi, Seungyeon, 5

Choi, Sunghoon, 51

Choi, Young-Wook, 51

Christensen, Søren, 17

Clackdoyle, Rolf, 3P

Clark, D. P., OA, OF, 11

Cobos, Santiago F., 28

Cockmartin, Lesley, OJ, OK

Colbeth, Richard E., 20, 3Q, 3W

Collins, J., 3B, 4Q

Conant, Emily F., OI

Cruz, Orquídea, 5J

Cruz-Bastida, Juan Pablo, 22

Curran, Walter J., 47, 48, 49, 4L, 4M, 4P

Daly, Megan, 31

Dance, David R., OR

Dangelmaier, Julia, $1 Q$

Danielsson, Mats, $\mathrm{OE}$

Das, Mini, 1C, 1G, 5B

De Brucker, Yannick, 15

De Man, Bruno, $1 \mathrm{H}$

de Mey, Johan, 15, 30

Deng, Xiaolei, 07, 41

De Paepe, Lode, 32

Desbat, Laurent, 3P 
Dewaraja, Yuni K., OU

Dey, Joyoni, $4 Z$

di Franco, Francesca, 59

Divel, Sarah E., 17

D'Moore, Desirée, 55

Dong, Xue, 47, 48, 49, 4P

Dow, Scott, OX

Doyle, Scott, 31

Duan, Jiayu, 30

Duan, Xiaoyu, $\mathrm{OH}, 0 \mathrm{O}$

Dustler, Magnus, 57, 58

Edic, Peter M., $1 \mathrm{H}$

Ehtiati, T., 1M

Elangovan, Premkumar, OR

El-Mohri, Youcef, 10

Engel, Klaus Juergen, 10, 34

Espy, Michelle, 3H

Eulig, Elias, 1L

Fang, Chengyijue, 3S, 56

Fang, Yuan, OG

Feigin-Almon, Micha, 2G

Feng, Mang, $1 \mathrm{~J}$

Fessler, Jeffrey A., OQ, OU

Figl, Michael, OK

Fingerle, Alexander A., $1 Q$

Fletcher, Joel G., 1R

Flohr, Thomas, IR

Fokuhl, Julia, 1Q

Fraley, David, 40

François, Christopher, 22

Fredette, Nathaniel R., $1 \mathrm{G}$

Freeman, Matthew S., $3 \mathrm{H}$

Freestone, Steven, 3W

Fricks, Rafael B., $2 Q$

Friel, Harry, 33

Frysch, Robert, 2S, 3J

Fu, Lin, $1 \mathrm{H}$

Fu, Shuai, 3T

Fu, Wanyi, 14, $1 \mathrm{U}$

Fujiwara, Hisao, 11

Fung, George S. K., 02

Gaalaas, Laurence, 2R

Gang, Grace J., OP, 18, 1Z, 27, 3Q

Gao, Hewei, 08, 1A, 1P

Gao, Mingjie, $0 Q$

Gao, Qi, OB, 42, 43, 44, 4B, 4D, 4U

Gao, Yongfeng, 21, 4A

Garrett, John W., 1K, 3G, 4H

Ge, Yongshuai, 07, 1E, 41, 4C

Gershenson, J., 5E

Gershenson, M., 5E

Ghammraoui, Bahaa, 0M, 4Y

Ghani, M., 52

Ghavidel, Beth, 4L, 4M

Gifford, Howard C., OT

Gilat Schmidt, Taly, 1F, 40

Glick, Stephen J., OM, 4Y

Go, Eunsol, 3F

Goldan, A., OX
Gomi, Tsutomu, 4F

Gong, Hao, 09

González, Francisco J., 5J

González, Raymundo, 5J

Graff, Christian G., OM

Gregory, Bradley, 52

Griner, Dalton, 4H

Guo, Chao, 3A, 3B, 3C, 3D, 3E

Guo, Hongbo, 2H

Guo, Jinchuan, $1 \mathrm{E}$

Guo, Zijia, 03

Gupta, Amar Prasad, 2E, 2V, 5C

Gupta, Ashish, OW

$\mathrm{Ha}$, Su Min, 5D

Haase, V., IN

Hahn, K., IN

Halaweish, Ahmed, IR

Hammoud, Dima, 33

Han, R., 26

Hao, You, 2K

Hara, Hidetake, 4F

Harmon, Ian E., 1C

Harms, Joseph, 4L, 4M

Harvey, Evan, 36

Hassan, Laila, 53

Haupt, Robert W., 2G

$\mathrm{He}$, Xiaowei, $2 \mathrm{H}$

Hellgren, Gustav, 58

Heo, Changyong, $2 U$

Herbst, M., 05

Hernandez, Andrew M., OL

Hertz, Hans M., $1 \mathrm{~V}$

Higgins, Kristin, 47, 48, 49

Hizukuri, Akiyoshi, 46

Hoeschen, Christoph, 2N

Holbrook, M. D., OF, 11

Holdsworth, David W., 28

Hong, Eunyeong, 4E

Hong, Zixuan, 3T

Hörndler, Klaus, $1 \mathrm{~L}$

Hou, Yuqing, 2H

Howansky, Adrian, OX

Hoye, Jocelyn, 20

$\mathrm{Hu}$, Zhenhua, $2 \mathrm{~F}$

Huang, Bangyan, 3K

Huang, Chongfei, 3L

Huang, Hailiang, $\mathrm{OH}, \mathrm{OO}$

Huang, Haiyun, 43, 4D

Huang, Jing, OB, 42, 43, 4D, 4U

Huck, Sascha Manuel, 02

Hughes, Julie M., 2G

Hummel, Johann, OK

Ichikawa, Yasutaka, 46

Inglis, Stuart, 31

Inscoe, Christina R., 04, 24, 2R, 37, 5F

Ionita, Ciprian N., 35

Ishida, Masaki, 46

Ishihara, Nozomi, $2 Z$

Izawa, Takashi, OD

xviii 
Jacobs, R., 2T

Jang, Hyunsuk, OG Jang, Jaekyu, 2V, 5C Jeon, Hyojin, 3F Jeong, Changwon, 2E Jeong, Jin-Woo, 3F $\mathrm{Ji}, \mathrm{XU}, 1 \mathrm{~B}, 1 \mathrm{~J}$ Jiang, Xiao, 3K, 56 Jiang, Xiaojun, 4P Jo, Mijung, 5D Job, Isaias, 3W Jones, Elizabeth, $1 Y$ Jordan, Petr, 40 Jung, Jaeik, 2E, 2V, 5C Jung, Kahee, 5D Kabir, M. Z., OG Kachelrieß, Marc, $1 \mathrm{~L}$ Kang, Jun-Tae, 3F Kang, Songhee, 4E Kanwal, Bushra, 55 Kapadia, Anuj, $1 T$ Kappler, S., 05 Karim, Karim S., 0G, 51 Kato, Munetaka, 11 Keelson, Benymeen, 30 Kessener, Yves, $3 Z$ Kim, Burnyoung, 2D, 45, 4I Kim, Dohyeon, 4S, 5A Kim, Dong Sik, 4E Kim, Dongkeun, 2E Kim, Eun Sil, 5D Kim, Hee-Joung, 4S, 5A, 51 Kim, Hyemi, 4S, 5A Kim, Jae-Woo, 3F Kim, Jong Hyo, $2 \mathrm{U}$ Kim, Namkug, 5C Kim, Seung Hoon, 5C Kim, Sihwan, $2 U$ Kim, Soo-Yeon, 5D Kim, Sunghee, 3F Kim, Wooseob, 2V Kimpe, Tom, 32 Kitagawa, Kakuya, 46 Kleinszig, G., 26 Knaup, Michael, $1 \mathrm{~L}$ Ko, Beomseok, 5C Kojima, Shinichi, 4V Kopp, Felix K., 1Q Kreher, B., 26 Krol, Andrzej, OT Krumina, Gunta, 2J Kulvait, Vojtěch, 3J Kumagai, Oichi, OD Kunze, H., 26 Laeseke, Paul F., 16, IW Lalonde, Robert, 4T Lansberg, Maarten G., 17 Larsson, Jakob C., IV Lauritsch, Günter, 03
Laux, Joseph, 33

LeClair, R. J., $3 Y$

Lee, Denny, OG

Lee, Do II, $2 U$

Lee, Eunae, 4E

Lee, Jeong-Woong, 3F

Lee, Ji Hyun, 33

Lee, Minjae, 4S, 5A

Lee, Seungwan, 2D, 45, 4I

Lee, Su Hyun, 5D

Lee, Youngjin, 2D

Lee, Yueh Z., 04, 24, 37, 38, 5F

Lei, Yang, 47, 48, 49, 4L, 4M, 4P

Leiner, Tim, 15

Leng, Shuai, $4 R$

Leong, Andrew F. T., OP

Léveillé, Sébastien, $0 X$

Lewis, Steven, 31

Li, Danyang, OB, 42, 43, 44, 4B, 4D, 4U

Li, Hua, 2K

$\mathrm{Li}$, Jing, $3 \mathrm{~N}$

$\mathrm{Li}, \mathrm{Ke}, \mathrm{OZ}, 1 \mathrm{~B}, 1 \mathrm{~J}, 1 \mathrm{~K}, 22,36,3 \mathrm{G}, 4 \mathrm{H}$

Li, Sui, OB, 42, 43, 44, 4B, 4D, 4U

Li, Taotao, 3R

Li, Xinbin, IA

Li, Y., 52

Li, Yang, 2B, 3L, 30

Li, Yi, 3N

Li, Yinsheng, 06, 1K, 22, 3G, 4H

Lian, Maosen, $3 \mathrm{~N}$

Liang, Albert K., 10

Liang, Dong, 07, 1E, 41, 4C

Liang, Kaichao, 3M

Liang, Zhengrong, 21, 4A

Liapi, Eleni, 18

Liduma, Sanita, 2J

Lim, Hongki, OU

Lin, Liyong, $4 \mathrm{M}$

Lippert, Thomas, 39

Liu, H., 52

Liu, Peizhen, $1 \mathrm{E}$

Liu, Ruirui, 15

Liu, Tian, 47, 48, 49, 4L, 4M, 4P

Liu, Yanqiu, $2 \mathrm{H}$

Liu, Yingzi, 4M, 4P

Lloyd, Christopher W., $2 \mathrm{G}$

LO, Joseph Y., OJ, 1T, 2 Y

Lo, S.-F., 26

Lohr, Rhiannon L., 51

Long, Wenting, 4W, $4 \mathrm{X}$

LU, Jianping, 04, 24, 2R, 37, 38, 5F

LU, Minghui, 20, 25, 3Q

Lu, Siming, 4A

Lubinsky, A. R., OX

Lubner, Meghan, 22

Luckner, C., 05

Luguzis, Artis, 2J

Luo, Shouhua, 3N

Luo, Yueting, 37, 38 
Lyu, Qihui, 4J

Lyu, Su Hyun, OL

Ma, Jianhua, OB, 3T, 42, 43, 44, 4B, 4D, 4U

Ma, Yiqun, $3 Q$

MacDonald, Carolyn A., 53, 55

Maes, Willy H., $3 Z$

Magnelind, Per E., 3H

Maidment, Andrew D. A., OI, 1Q, 32, 57, 5G

Maier, Andreas, 03, 1N

Maier, Joscha, $1 \mathrm{~L}$

Makeev, Andrey, 0M, 4Y

Mao, Hui, 47, 48, 49

Marsh, Jeffrey, Jr., 4R

Marshall, Matthew S. J., 12

Marshall, N. W., OJ

Mativenga, Mallory, 2E

Matsumoto, Isao, $2 \mathrm{Z}$

Mazurowski, Maciej, $1 \mathrm{~T}$

McCarthy, E. L., 3Y

McCollough, Cynthia H., 09, 1R, 4R

McDonald, Mark, 4L

McDonough, Joseph M., 2K

Medrano, Maria, is

Meng, Deyu, OB, 44

Meng, Mingqiang, OB, 42, 44

Menser, Bernd, 10, 34

Merken, K., 2T

Merrill, Frank E., 3H

Mettivier, Giovanni, 59

Miller, James J., $3 Z$

Miller, Jessica R., $1 \mathrm{~J}$

Miller, Stuart R., 12

Mishchenko, Anastasiia, OX

Mizukami, Shinya, 4F

Mohan, Chandra, $1 G$

Montoya, Juan, $3 G$

Mou, Xuanqin, 21, 2B, 3L, 30

$\mathrm{Na}$, Yong Ju, 5D

Nagarkar, Vivek V., 12

Nagata, Motonori, 46

Nagi, Chandandeep, 5B

Nakamura, Tadashi, 2C

Nakayama, Ryohei, 46

Navab, Nassir, 1Q

$\mathrm{Ng}$, Susan, 57

Nguyen, Hung, 3P

$\mathrm{Ni}$, Yifan, $1 \mathrm{E}$

Nikolau, Ethan P., 1 W

Nikolov, Hristo N., 28

Nishihara, Toshiyuki, OD

Nissman, Daniel, 04

Niu, Tianye, 4J

Noël, Peter B., OI, 18, 1Q, $5 G$

Noo, Frédéric, 03, $1 \mathrm{~N}$

Nye, Jonathon A., 47, 48, 49

Oberstar, Erick L., 16

Offe, Michael, 40

Okada, Yoshihiro, 11
Omoumi, F., 52

Ong, Chloe Leigh, $\mathrm{OH}$

O'Sullivan, Joseph A., is

Pahn, Gregor, 1Q

Park, Junyoung, 2E

Park, Sora, 3F

Park, Yeonok, 2W

Parodi, Katia, 02

Partain, Larry, 31

Passand, Zahra, 2N

Pegues, Hope, $2 \mathrm{Y}$

Pelc, Norbert J., 17, 1P

Periyasamy, Sarvesh, 16, 1W

Perrone, Lisa, 2R

Persson, Mats U., OE, $1 \mathrm{H}$

Peters, Inge M., $3 Z$

Petrov, Dimitar, OK

Petruccelli, Jonathan C., 53, 55

Pfeiffer, Daniela, 1Q

Pham, Thahn Tra, 58

Philips, Wilfried, 32

Pil-Ali, Abdollah, 51

Platisa, Ljiljana, 32

Plies, Michael, 31

Podgorsak, A., 4Q

Politis, C., 2T

Politte, David G., 15

Pollmann, Steven I., 28

Porras-Chaverri, Mariela, is

Portero Lopez, Violeta, 30

Principi, Sara, 40

Puett, Connor, 04, 24, 2R, 5F

Punzet, Daniel, 2S, 3J

Pyakurel, Uttam, 55

Rajagopal, Jayasai, IY

Rajendran, Kishore, IR, 4R

Rava, Ryan A., 35

Regan Anderson, Michael, 2R

Ren, Liqiang, 09, $1 \mathrm{R}$

Ren, Yan, 4W, 4X

Renforth, Kate L., OR

Renger, Bernhard, 1Q

Ria, Francesco, 14

Richards, Taylor, 20

Richardson, Jonathan M., $2 G$

Ríos, Julián, 5J

Ritman, Erik, 4R

Ritschl, L., 05

Rodriguez Luna, J. C., 5B

Rohr, Pierre, 10

Rojas, Oscar, 33

Rose, Georg, 2S, 3J

Ruan, Dan, 4J

Rudin, Stephen, OY, 35, 3A, 3B, 3C, 3D, 3E, 4Q

Ruetten, Walter, 10, 34

Rummeny, Ernst J., $1 Q$

Russo, Paolo, 59

Ryu, Jehwang, 2E, 2V, 5C

Saad, Fatima, 3J 
Sadri, Amir Reza, OW

Sakuma, Hajime, 46

Salomon, Elisabeth, OK

Samala, Ravi K., $O Q$

Samei, Ehsan, 14, 1T, 1U, 1Y, 2O, 2Q, 2Y, $2 Z$

Sarno, Antonio, 59

Saver, Thomas, $1 \mathrm{U}, 1 \mathrm{Y}$

Sauter, Andreas P., 1Q

Schaefer, Dirk, 34

Scheerlink, Thierry, 30

Schevermann, James, OX

Schmidt, Bernhard, $1 \mathrm{R}$

Schmidtlein, C. Ross, OT

Schmitz, Daniel, 39

Schöndube, H., IN

Schultheiß, Manuel, 1Q

Scott, Chris C., 51

Segars, William Paul, 1T, 1Y, $2 Z$

Semturs, Friedrich, OK

Seo, Wontaek, 5D

Setiawan, Hananiel, 14

Setlur Nagesh, S. V., OY

Shaheen, E., 2T

Shaker, Kian, IV

Shapira, Nadav, $1 Q$

Shapiro, Edward, 20

Shen, Liuxing, 10

Sheng, Ke, 4J

Sheth, Niral, 12, 26

Shi, Gengxin, 12

Shi, Linxi, 20, 25, 2M

Shi, Yongyi, 21, 4A

Shin, Choul Woo, 5D

Shinohara, Norimitsu, OD

Shu, Hui-Kuo, 4P

Sidebottom, Rachel B., 3H

Sidky, Emil Y., $1 \mathrm{~F}$

Siewerdsen, Jeffrey H., 05, 12, 1M, 26, 27

Simon, Matthias, 10, 34

Singh, Bipin, 12

Sisniega, Alejandro, OP, 1M, 26

Smith, Bryce, $4 Z$

Smith, Taylor Brunton, 14, 10

Soh, Jeongtae, 2E

Solomon, Jeffrey, 33

Solomon, Justin B., 1U, 2O, 2Q

Soloviev, Vadim Y., OR

Song, Samuel, 31

Song, Yoon-Ho, 3F

Speck, Oliver, 25

Speidel, Michael A., 16, 1W

Spronk, Derrek, 37, 38

Stalbaum, Tyler, 31

Star-Lack, Josh, 20, 25, 3Q

Stavro, Jann, OX

Stayman, J. Webster, OP, 18, 1M, 1Z, 27, 3Q

Stierstorfer, Karl, 02, 1N

Stokes, William, 4L

Strother, Charles, 1K, 3G
Su, Ting, 07, 41, 4C

Sun, Sheng-Hsuan, 3A, 3E

Sun, Weiyuan, 53

Sun, Xindong, 07, 41

Sundberg, Christel, OE

Taguchi, Katsuyuki, 0C

Tai, Ya-Hsiang, $3 \mathrm{X}$

Tajima, Takashi, 11

Takahashi, Isao, 4V

Takahashi, Keiko, 2C

Tamura, Masaya, $2 Z$

Tan, Jiaxing, 4A

Tanaka, Rie, $2 Z$

Tang, Xiangyang, 4P, 4W, 4X

Tanguay, Jesse, 4T

Tanimoto, Tatsunori, 11

Tao, Shengzhen, 4R

Tao, Xi, 3T

Taskin, Ulas, $5 \mathrm{H}$

Theodore, N., 26

Thomasson, David, 33

Thran, Axel, 10, 34

Tian, Jie, $2 \mathrm{~F}$

Tian, Yi, 2X, 3R

Tian, Zhen, 4P

Tingberg, Anders, 57, 58

Tivnan, Matthew, 18, 1Z, 3Q

Tiwari, Pallavi, OW

Tognina, Carlo, $3 \mathrm{~W}$

Tong, Huayu, $2 Y$

Tong, Yubing, 2K

Torigian, Drew A., 2K

Torres, Luis A., IW

Toumi, Mehdi, 50

Tousignant, Olivier, $3 z$

Troville, Jonathan, 3B, 3C, 3D, 3E, 4Q

Tupa, Dale, 3H

Udupa, Jayaram K., 2K

Uneri, A., 26

Unger, Ewald, OK

Ushikura, Shinichi, 11

Vagdargi, P., 26

Vancoillie, Liesbeth, 0J, OK

Vandemeulebroucke, Jef, 15, 30

van Dongen, Koen W. A., $5 \mathrm{H}$

Van Gompel, Gert, 15, 30

Vasudev, Varun, 32

Vazquez, Ivan, 1C

Vent, Trevor L., OI, 5G

Vercnocke, Andrew, 4R

Vijayan, R., 26

Viswanath, Satish E., OW

Vogt, S., 05, 26

Vullers, Ruud J. M., $3 Z$

Wagner, Martin G., IW

Wainman, Bruce, 31

Wang, Adam S., 1L, 1P, 20, 25, 2M, 40

Wang, Lei, 42, 44

Wang, Tianshu, $3 \mathrm{~N}$ 
Wang, Tonghe, 47, 48, 49, 4L, 4M, 4P

Wang, Wenying, OP, 18, 1Z, 3Q

Wang, Yang, $2 X$

Wang, Yongbo, 3T

Watanabe, Yusuke, 4F

Webb, Tyler, is

Weil, Michael, 31

Weisfield, Richard, 3W

Weiss, C. R., 1M

Wells, Kevin, OR

Wells, Stephen, OR

Wen, Xiang, 3R

Whitehead, Joseph F., IW

Whiting, Bruce, is

Wikner, J. Jacob, OE

Wiley, Benjamin J., $2 Y$

Wileyto, E. Paul, 5G

Williamson, Jeffrey, 15

Wong, M., 52

Wu, Caiyun, 2K

Wu, Chengpeng, 1A

Wu, Chengyang, 40

Wu, Jesse, OP

Wu, P., 26

Wu, X., 52

Wu, Yijing, 3G

Xie, Huiqiao, 4W, 4X

Xie, Qi, OB, 44

Xing, Yuxiang, 08, 3M

$X u$, Gongming, 3S, 56

$X \cup$, Jingzhu, $4 Z$

$\mathrm{Xu}$, Yan, 40

$\mathrm{Xu}$, Yuesheng, OT

Yamakawa, Keisuke, $2 \mathrm{C}$

Yamashiro, Tsuneo, $2 Z$

Yang, Xiaofeng, 47, 48, 49, 4L, 4M, 4P, 4X

Yang, Yirong, 3M

Yao, Jingwu, 40

Yao, Lisha, OB, 4B, 4U

Yeh, Shan, 3X

Yeo, Seung Jun, 5C

Yeon, Ji-Hwan, 3F

Yi, Ann, 5D

Yim, Dobin, 2D, 45, 4I

Yokoi, Kazuma, 4V

Yoo, Namjo, 4E

Yoon, Kwon-Ha, 2E

Young, Kenneth C., OR

Yu, Lifeng, 09, 1R

Yu, Zhelin, 2W

Yu, Zhou, 23

Yun, Ki Nam, 3F

Zackrisson, Sophia, 57

Zbijewski, Wojciech, 05, 12, 1M, 3Q

Zeng, Dong, OB, 42, 43, 44, 4B, 4D, 4U

Zhang, Chengzhu, 06

Zhang, G., 2T

Zhang, Guoqing, 2X, 3R

Zhang, Hua, 3T
Zhang, Jun, $4 \mathrm{~N}$

Zhang, Li, 08, 1A

Zhang, Pu, 40

Zhang, Ran, 1B, $1 \mathrm{~J}$

Zhang, Ruoqiao, 23

Zhang, Shanli, 43, 4D, 4U

Zhang, Xiaoyun, 55

Zhang, Xinyu, 42, 4B

Zhang, Yikun, 4C

Zhao, C., 05

Zhao, Hengna, 2H

Zhao, Qian, OB, 44

Zhao, Qihua, 10

Zhao, Tianyu, is

Zhao, Wei, $\mathrm{OH}, 0 \mathrm{O}, \mathrm{OX}$

Zheng, Ao, 08

Zheng, B., 52

Zheng, Hairong, 07, 41, 4C

Zhi, Shaohua, 2B, 3L, 30

Zhong, Rikui, 4B

Zhou, Hao, IP

Zhou, Jian, 23

Zhou, Jun, 4L, 4M

Zhou, Otto Z., 04, 24, 2R, 37, 38, 5F

Zhou, Wei, 50

Zhou, Weimin, OV

Zhu, Jiongtao, 41, 4C

Zhu, Lei, 1P, 3K, 3S, 56

Zhu, Manman, OB, 42, 43, 44, 4B, 4D, 4U

Zhu, Xiaohua, 1A

Ziskin, Vitaliy, 31

Zuo, Hongquan, 4N

xxii

Proc. of SPIE Vol. 11312 1131201-22 


\section{Conference Committee}

Symposium Chairs

Georgia D. Tourassi, Oak Ridge National Laboratory (United States)

Metin N. Gurcan, M.D., Wake Forest Baptist Medical Center

(United States)

Conference Chairs

Guang-Hong Chen, University of Wisconsin School of Medicine and

Public Health (United States)

Hilde Bosmans, UZ Leuven (Belgium)

Conference Co-chair

Wei Zhao, Stony Brook Medicine (United States)

Conference Program Committee

Shiva Abbaszadeh, University of Illinois at Urbana-Champaign (United States)

Adam M. Alessio, Michigan State University (United States)

Mini Das, University of Houston (United States)

Mats E. Danielsson, KTH Royal Institute of Technology (Sweden)

Maria Drangova, Robarts Research Institute (Canada)

Rebecca Fahrig, Siemens Healthineers (Germany)

Thomas G. Flohr, Siemens Healthineers (Germany)

Arundhuti Ganguly, Varex Imaging Corporation (United States)

Yongshuai Ge, Shenzhen Institutes of Advanced Technology (China)

Taly Gilat Schmidt, Marquette University (United States)

Stephen J. Glick, U.S. Food and Drug Administration (United States)

and University of Massachusetts Medical School (United States)

Marc Kachelriess, Deutsches Krebsforschungszentrum (Germany)

Karim S. Karim, University of Waterloo (Canada)

Hee-Joung Kim, Yonsei University (Korea, Republic of)

Patrick J. La Rivière, The University of Chicago (United States)

Quanzheng Li, Massachusetts General Hospital (United States)

Joseph Yuan-Chieh Lo, Carl E. Ravin Advanced Imaging

Laboratories, Duke University (United States)

Peter B. Noël, University of Pennsylvania (United States)

Frédéric Noo, The University of Utah (United States)

Jinyi Qi, University of California, Davis (United States)

John M. Sabol, GE Healthcare (United States) 
loannis Sechopoulos, Radboud University Medical Center

(Netherlands)

Joseph W. Stayman, Johns Hopkins University (United States)

Anders Tingberg, Lund University (Sweden)

Adam S. Wang, Stanford University School of Medicine (United States)

Yuxiang Xing, Tsinghua University (China)

John I. Yorkston, Carestream Health, Inc. (United States)

Lifeng Yu, Mayo Clinic (United States)

Behrouz Shabestari, National Institute of Biomedical Imaging and Bioengineering (United States)

\section{Session Chairs}

1 New Technologies in Imaging

Wei Zhao, Stony Brook Medicine (United States)

Guang-Hong Chen, University of Wisconsin School of Medicine and Public Health (United States)

2 CT Reconstruction with DL Techniques

Marc Kachelriess, Deutsches Krebsforschungszentrum (Germany)

Jinyi Qi, University of California, Davis (United States)

3 Photon Counting Detectors

Mats E. Danielsson, KTH Royal Institute of Technology (Sweden)

Stephen J. Glick U.S. Food and Drug Administration (United States) and University of Massachusetts Medical School (United States)

4 Quality Assessment and Optimization in Breast Imaging

Hilde Bosmans, UZ Leuven (Belgium)

Joseph Yuan-Chieh Lo, Carl E. Ravin Advanced Imaging Laboratories, Duke University (United States)

5 Breast Imaging: New Technology

Patrick J. La Rivière, The University of Chicago (United States)

Hee-Joung Kim, Yonsei University (Korea, Republic of)

6 Innovations in Nuclear Medicine and MRI

Guang-Hong Chen, University of Wisconsin School of Medicine and Public Health (United States)

Quanzheng Li, Massachusetts General Hospital (United States)

7 Detector Technologies

Rebecca Fahrig, Siemens Healthineers (Germany)

Karim S. Karim, University of Waterloo (Canada) 
8 Target: Contrast Agent Imaging Optimized

John I. Yorkston, Carestream Health, Inc. (United States)

Maria Drangova, Robarts Research Institute (Canada)

$9 \quad$ X-ray Phase Contrast Imaging

Peter B. Noël, University of Pennsylvania (United States)

Mini Das, University of Houston (United States)

10 Photon Counting CT Applications

Lifeng Yu, Mayo Clinic (United States)

Arundhuti Ganguly, Varex Imaging Corporation (United States)

11 CBCT Reconstruction Techniques

Adam M. Alessio, Michigan State University (United States)

Adam S. Wang, Stanford University School of Medicine (United States)

12 Spectral CT New Hardware

John M. Sabol, GE Healthcare (United States)

Taly Gilat Schmidt, Marquette University (United States)

13 Virtual Imaging

Ioannis Sechopoulos, Radboud University Medical Center (Netherlands)

Joseph Yuan-Chieh Lo, Carl E. Ravin Advanced Imaging Laboratories, Duke University (United States)

14 New Reconstruction Techniques in Dual Energy CT

Frédéric Noo, The University of Utah (United States)

15 Artifacts in CBCT Solved

Joseph W. Stayman, Johns Hopkins University (United States)

Lifeng Yu, Mayo Clinic (United States) 
Proc. of SPIE Vol. 11312 1131201-26

Downloaded From: https://www.spiedigitallibrary.org/conference-proceedings-of-spie on 25 Apr 2023 Terms of Use: https://www.spiedigitallibrary.org/terms-of-use 


\section{Medical Imaging Award Recipients}

\section{Robert F. Wagner Best Student Paper Award}

Robert F. Wagner was an active scientist in the SPIE Medical Imaging meeting, starting with the first meeting in 1972 and continuing throughout his career. He ensured that the BRH, and subsequently the CDRH, was a sponsor for the early and subsequent Medical Imaging meetings, helping to launch and ensure the historical success of the meeting. The Robert F. Wagner All-Conference Best Student Paper Award (established 2014) is acknowledgment of his many important contributions to the Medical Imaging meeting and his many important advances to the field of medical imaging.

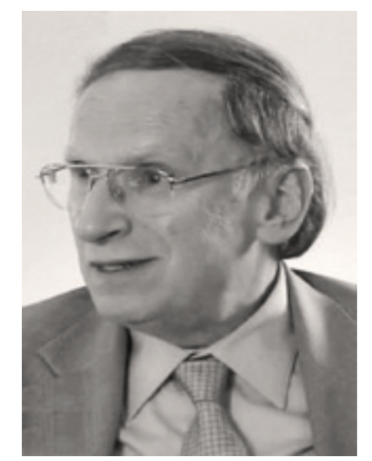

This award is co-sponsored by:

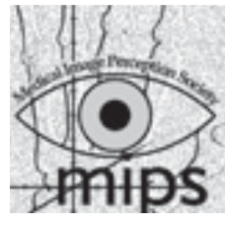

The Medical Image Perception Society

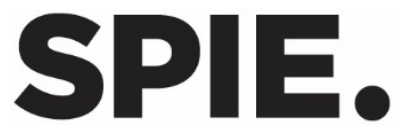

2020 Recipients:

First Place: Multi-body registration for fracture reduction in orthopaedic trauma surgery (11315-14)

R. Han, A. Uneri, P. Wu, R. Vijayan, P. Vagdargi, M. Ketcha, N. Sheth, Johns Hopkins University (United States), S. Vogt, G. Kleinszig, Siemens Healthineers (Germany) G. M. Osgood, John Hopkins Hospital (United States), J. H. Siewerdsen, John Hopkins University (United States)

Second Place: Phase contrast CT enabled three-material decomposition in spectral CT imaging (11312-47)

Xu Ji, Ran Zhang, Ke Li, Guang-Hong Chen, University of Wisconsin School of Medicine and Public Health (United States)

\section{Physics of Medical Imaging Student Paper Awards sponsored by GE Healthcare}

Winner: Phase contrast CT enabled three-material decomposition in spectral CT imaging (11312-47)

XU Ji, Ran Zhang, Ke Li, Guang-Hong Chen, University of Wisconsin School of Medicine and Public Health (United States)

Runner-up: Method for metal artifact avoidance in C-Arm cone-beam CT (11312-78) P. Wu, N. Sheth, A. Sisniega, A. Uneri, R. Han, R. Vijayan, P. Vagdargi, Johns Hopkins University (United States), B. Kreher, H. Kunze, G. Kleinszig, S. Vogt, Siemens Healthineers (Germany),

S.-F. Lo, N. Theodore, and J. H. Siewerdsen, John Hopkins University (United States) 
Runner-up: $1 \boldsymbol{\mu m}$ spatial resolution in silicon photon-counting CT detectors by measuring charge diffusion (11312-14)

Christel Sundberg, Mats Persson, KTH Royal Institute of Technology (Sweden), J. Jacob Wikner, Linköping University, (Sweden) Mats Danielsson, KTH Royal Institute of Technology (Sweden) 